\title{
La reflexividad etnográfica como soporte investigativo en las acciones publicitarias*
}

\author{
Zulima Azeneth López Torres* \\ Isabel Cristina Torres Estrada*** \\ Recibido: Enero 15 - Aprobado: Marzo 15
}

Con las comunicaciones expandidas y las influencias interculturales, la gente interpreta a los otros, y se interpreta a sí misma, en una pasmosa diversidad de idiomas [...] este mundo ambiguo y multívoco hace que cada vez resulte más difícil concebir la diversidad humana como algo inscrito en culturas cerradas e independientes.

(James Clifford)

\section{Resumen}

Este artículo busca exponer la importancia de la reflexividad etnográfica como una herramienta investigativa que puede posicionarse como una de las mejores estrategias para el abordaje del consumidor, en tanto el sujeto es atravesado por el devenir de su realidad social. La etnografía proporciona una visión holística de ese sujeto que consume, en relación consigo mismo, con el entorno y con los objetos y/o bienes disponibles para la satisfacción de sus necesidades, en tanto que permite un análisis de los procesos mercantiles o no, de los que disponen los miembros de una sociedad, que a su vez se convierten en configuradores de representaciones sociales e imaginarios en relación con las adquisiciones, usos y adhesiones, entre otras cosas de lo que en este texto se ha denominado como mercancías. Estas posibilidades investigativas le proponen al ejercicio publicitario hallar, dentro del cúmulo de datos cotidianos, los elementos comunes que subyacen en las configuraciones sociales, buscando que la retórica en este tipo de comunicación sea acorde con estas, a fin de cumplir su fin último: la persuasión.

Palabras clave: etnografía, antropología, consumidor, publicidad, investigación, mercancías, realidad social, EMIC, ETIC

* $\quad$ Este artículo de carácter reflexivo surge a partir de los resultados arrojados del macroproyecto de investigación denominado "Fundamentación teórica del concepto de comportamiento del consumidor desde la economía, la antropología, la sociología, la psicología y la estética", el cual tuvo financiación por parte de la Universidad Pontificia Bolivariana para su desarrollo.

** Docente. Coordinadora línea Consumo y Entorno del grupo de investigación Epilión. Coordinadora Académica de la Maestría en Comportamiento del Consumidor. Facultad de Publicidad, Escuela de Ciencias Sociales, Universidad Pontificia Bolivariana. Dirección electrónica: zulima.lopez@upb.edu.co

*** Docente. Coordinadora del grupo de investigación Epilión. Facultad de Publicidad, Escuela de Ciencias Sociales. Dirección electrónica: Isabel.torres@upb.edu.co 


\title{
Ethnographic Reflexivity as Research Support in Advertising
}

\begin{abstract}
This article is intended to express the importance of ethnographic reflexivity as a research tool which can be positioned itself as one of the best strategies to address consumers, since individuals are influenced by the course of their social reality. Ethnography provides a holistic point of view of those persons who consume with respect to themselves, the environment, and the objects and/or goods available for meeting their needs, since it allows making an analysis of the business processes available for the members of a society who in turn become configurators of social representations and imaginaries in relation to acquisitions, uses, and adhesions, among other things, of what has been called merchandise in this article. These research possibilities allow the advertising exercise to find, among the number of daily data, the common elements underlying social configurations so that rhetoric in this kind of communication can be consistent with them in an attempt to reach the goal: persuasion.
\end{abstract}

Key words: Ethnography; anthropology; consumer; advertising; research; merchandise; social reality; EMIC; ETIC. 


\section{Metodología}

El presente artículo es una reflexión derivada de los resultados arrojados por la investigación de carácter cualitativo, sobre los aportes teóricos que desde diferentes disciplinas sociales se han hecho a la fundamentación del comportamiento del consumidor, específicamente desde la antropología y su método de investigación por excelencia: la etnografía, en relación con la publicidad y el mercadeo.

Dicha investigación fue realizada a partir de revisión documental y de entrevista semiestructurada a expertos inscritos en las disciplinas de antropología, psicología, economía, estética y sociología, vinculados profesionalmente a los procesos de mercadeo y a la planeación publicitaria en diferentes empresas, a fin de comprender la manera como dichas disciplinas hacen parte de estos procesos. La muestra estuvo determinada por el principio de saturación teórica, y el enfoque de análisis fue el etnográfico, de tal manera que se pudiese hacer una triangulación entre los datos recogidos, la teoría construida y la interpretación propia de los investigadores.

\section{Introducción}

\section{La etnografía, una manera de comprender e interpretar las "Otras" realidades}

\section{Breve recuento histórico}

Es sabido ya, que desde la Grecia antigua había un interés marcado por la "Otredad", conformada por todos aquellos que no tenían las costumbres griegas, pero sobre todo por aquellos que no hablaban su misma lengua. Uno de los primeros interesados en describir, de manera rigurosa, esos pueblos no griegos que representaban una dosis de exotismo era Herodoto; asimismo lo hizo Aristóteles, aunque desde una postura mucho más etnocéntrica que el primero, resaltando la superioridad helénica, con respecto a la inferioridad de los otros pueblos en tanto no habitantes de la "polis".

Tal interés descriptivo y comparativo (que, además, puede observarse en diversas crónicas e informes de conquistas, exploraciones, ocupaciones y demás momentos de encuentros entre 'civilizados' y 'primitivos' a lo largo de la historia), se considera como el antecedente de la etnografía que comienza a tomar forma con la llegada del Iluminismo "[...] y su apreciación acerca de los "otros" y la explicación acerca de lo que se consideraba como las distintas etapas evolutivas de la humanidad (salvajismo, barbarie, civilización)" (Ameigeiras, 2006, p. 110). Esta explicación evolucionista se consolida en las postrimerías del siglo XIX, por influencia de las ciencias naturales (especialmente la botánica y la zoología), pasando "[... a considerar la peculiaridad de las sociedades "primitivas" como sociedades ubicadas en una etapa evolutiva inferior" (Ameigeiras, 2006, p. 111), lo cual mantuvo ese etnocentrismo antes solo helénico, ahora europeo, pero que dio origen al objeto de estudio antropológico: la otredad o alteridad cultural.

Es hasta que aparece la crítica al "comparativismo evolucionista" planteada por Franz Boas (1858-1942), a partir de su escuela teórica denominada "particularismo histórico", 
que se comienza a abandonar esta postura etnocéntrica para, en su lugar, comprender y describir a cada sociedad desde sus configuraciones únicas y relativas. Boas hacía un llamado a una investigación "basada en la rigurosidad de los datos empíricos y en la consideración de la particularidad de los rasgos de cada cultura, que demandaba la presencia del investigador en el campo la fin] de no sacar rasgos fuera de contexto" (Ameigeiras, 2006, p. 111).

No obstante, tal concepción y abordaje investigativo de la "otredad" solo tuvo su reconocimiento y consolidación, a partir de lo planteado por Bronislaw Malinowski, quien encontró en lo que él denominó como "observación participante", la vía para esa rigurosidad que pedía Boas.

\section{- La revolución malinowskiana}

Bronislaw Malinowski (1884-1942) es considerado en la comunidad antropológica, sin lugar a dudas, como el gran transformador de la etnografía o, por lo menos, quien se encargó de su legitimación. Físico y químico de formación inicial, se acercó a la antropología por vía indirecta a partir de su encuentro con el libro La rama dorada (1890), escrito por J. G. Frazer (1854-1951), en el cual hace un proceso descriptivo-comparativo de la mitología y la religión "primitivas" de diversos pueblos. A partir de esto, Malinowski decide estudiar Antropología en la London School of Economics, donde conoce a su profesor C. G. Seligman quien le anima al trabajo de campo en Australia y Melanesia.

Las condiciones histórico-políticas del momento (estallido de la Primera Guerra Mundial) le llevan a su 'confinamiento' en estos lugares por un período de tiempo prolongado (aproximadamente 2 años y medio), período que se convirtió en un extenso trabajo de campo, en el que pudo realizar una detallada descripción de los habitantes de los archipiélagos de Nueva Guinea Oriental -los trobriand-, a partir de una institución social: la economía.

Fruto de tal investigación surge su libro más representativo: Argonautas del Pacífico occidental (1922), en el cual describe todo el complejo proceso de intercambio comercial de estos nativos, pero en el que, además, en su introducción, funda los elementos más importantes de la "nueva" investigación etnográfica:

[...] Malinowski no solo dio cuenta de un modo de describir una práctica extraña y, por ello, intraductible, adoptando en lo posible la perspectiva de los nativos; también hizo evidente la diferencia entre "describir" y "explicar", y los pasos necesarios para que una descripción no fuera invadida por la teoría y el mundo cultural del investigador (Guber, 2001, p. 30).

De tal postura, surgió el eje vertebrador de su propuesta etnográfica: la "observación participante", que se encuentra, además, atravesada por su concepción de la cultura como un sistema integrado (Malinowski, 2001) y que como tal, todo se encuentra relacionado con todo. Tal planteamiento lo fundamentó en el hecho de que "Hay toda una serie de fenómenos de gran importancia que no pueden recogerse mediante interrogatorios ni 
con el análisis de documentos, sino que tienen que ser observados en su plena realidad. Llamémosles los imponderables de la vida real" (Malinowski, 2001, p. 67). Esto es, el trabajo etnográfico tiene una finalidad y es que a partir de los esquemas que se construyan de la estructura social de un colectivo determinado, se deben extraer del cúmulo de hechos irrelevantes, aquellos elementos que se constituyen en las normas y leyes que rigen la vida cotidiana, pero que no son ni medibles, ni cuantificables, que operan de manera inconsciente y determinan los parámetros de comportamiento desde todo punto de vista.

Esta observación participante requiere de la presencia insustituible del investigador en el campo, en tanto que es la fuente fidedigna de información,

[...] pues solo "estando allí" podía el etnógrafo vincularse con ese pueblo, como un científico aborda el mundo natural [...] solo el trabajo de campo sin mediaciones podía garantizar la distinción entre cultura real y cultura ideal, entre lo que la gente hace y lo que la gente dice que hace, y por consiguiente, entre el campo de las prácticas y el de los valores y las normas (Guber, 2001, p. 33).

Esta certeza, aunque cuestionable (debido a la misma subjetividad del investigador) se convirtió en piedra angular para destacar la búsqueda de la imperturbabilidad de la vida cotidiana de quien se pretende investigar, a fin de garantizar la mayor objetividad en los procesos descriptivos e interpretativos.

Tal postura metodológica hizo que la antropología abandonase las cómodas instalaciones del 'gabinete' que hasta ahora ocupaba, para "aprender la racionalidad del indígena desde la vida diaria" (Guber, 2001, p. 33), lo cual marcó el desarrollo futuro de la disciplina misma y que posteriormente se extendió al resto de las ciencias sociales.

\section{- La reflexividad etnográfica}

El cuestionamiento profundo que se le hizo a esta postura metodológica surgió en la década de los cincuenta, a raíz de las propuestas que recién surgían tales como el estructuralismo de Claude Levi-Strauss (1908-2009) y el enfoque interpretativista o "etnografía simbólica" de Clifford Geertz (1926-2006). Esta crítica partía, esencialmente, de "[...] la necesidad de construir una etnografía tendente a desentrañar las estructuras de la significación, llevando a cabo la construcción de un tipo especial de descripción de carácter antropológico [...]" (Ameigeiras, 2006, p. 112). Tal necesidad puso en consideración lo que en adelante se denominó como "la reflexividad etnográfica".

Esto es, no es suficiente, tal como lo planteaba Malinowski, permanecer períodos prolongados de tiempo en el campo o terreno de investigación; ello permitía, sí, hacer un proceso descriptivo de la vida cotidiana de una comunidad, pero no se ahondaba en el significado y en los procesos reflexivos que se requerían para 'comprender' lo que esa comunidad vive; es decir, no había procesos realmente interpretativos de esas cotidianidades.

En este sentido, la 'reflexividad etnográfica', según Ameigeiras

[...] supone un replanteo de la forma y el modo de producir el conocimiento social, tomando distancia de posiciones positivistas como subjetivistas y asumiendo la 
capacidad reflexiva de los sujetos, que permite acceder a las interpretaciones acerca del mundo social en que se desenvuelve su existencia. Una reflexión que conduce a una revisión acerca del modo y la forma en que los sujetos producen el conocimiento social imprescindible para la coexistencia en sociedad. El punto de partida de la reflexividad implica considerar así al hombre como parte del mundo social, interactuando, observando y participando con otros hombres en un contexto y en una situación espaciotemporal determinada y, desde allí, considerar al propio investigador como parte del mundo que estudia (2006, p. 115).

Esto es, los sujetos se mueven en su mundo socialmente construido; tal construcción implica la creación de normas y leyes, dotadas de significados, a partir de las cuales se aprehende el mundo y, por ende, se mueve en su interior. Comprender e interpretar estas normas es la razón de ser del investigador porque

[...] describir y analizar el proceso social en su diversidad y singularidad implica rescatar la lógica de la producción material y simbólica de los sujetos sociales. En efecto, puesto en su tarea investigativa, el [investigador] se encuentra ante una determinada configuración histórica de acciones y nociones; solo dentro de ella, el mundo social cobra sentido para quienes lo producen y, a la vez, se reproducen en él (Guber, 2004, p. 73).

Así, el acto de un sujeto (sea este individual o colectivo) cobra sentido si este acto tiene una significación claramente determinada y asignada por el grupo en el cual se desarrolla. La tarea es entonces desentrañar tal significación, lo cual solo es posible si se triangulan las tres reflexividades que Guber (2001) propone, a saber: la reflexividad del investigador como miembro de una cultura determinada; la reflexividad del investigador como investigador -Lo ETIC- $-^{1}$ (teniendo en cuenta el constructo teórico desde el cual se apuntala, su comunidad académica, etc.) y finalmente, la reflexividad de la población que se busca estudiar -Lo EMIC-.

En este sentido, la etnografía se reconfigura y redefine, tal como lo plantea Spradley: "[...] Mas que estudiar a la gente, la etnografía significa aprender de la gente. El núcleo central de la etnografía es la preocupación por captar el significado de las acciones y los sucesos para la gente que tratamos de comprender" (1979, citado por Ameigeiras, 2006, p. 114).

\section{El encuentro publicidad-etnografía: ¿un matrimonio conveniente o una unión forzada?}

La publicidad emergió como discurso asociado a los avatares de la Modernidad y las transformaciones sociales y económicas que planteaba la llegada del capitalismo, de tal forma que

[...] permitió la incorporación de las diferentes clases sociales a una cultura de mercado. Esta cultura de mercado, a su vez, empleó una tecnocracia de la sensualidad cuya publicidad dio más importancia al valor de uso de la mercancía que al valor de cambio (Colón, 2001, p. 17).

1 Lo ETIC y lo EMIC: Conceptos que se desarrollan más adelante en relación con la publicidad 
En este orden, la concepción más concreta que se hace de la Publicidad, es la que plantea que esta es una comunicación con fines persuasivos; ello no es erróneo en absoluto, solo que en tal carácter persuasivo se encuentran contenidos diversos aspectos que la hacen sitio de confluencia de varias disciplinas y por ello mismo se le considera como Campo de Conocimiento.

Dichos aspectos, según Sánchez Guzmán (1993), se pueden condensar en tres bloques fundamentales: sus preguntas problematizadoras, su lenguaje y sus metodologías. Con respecto al primer bloque, es claro que las preguntas que se plantea la publicidad no le son de su propiedad y exclusividad, sino que son compartidas con otras disciplinas tales como la economía, la psicología, la semiología, la sociología, el derecho, la antropología, la historia y la comunicación. Por otra parte, el lenguaje utilizado desde y para la publicidad tampoco tiene dicho carácter de exclusividad y/o se considera como de carácter técnico. Finalmente, con respecto a lo metodológico, la publicidad hace uso de los procedimientos y herramientas que "[...] no son privativos a ella, porque trascienden a cualquier disciplina" (Sánchez, 1993, p. 33).

Como ya se entiende, por el texto introductorio del presente artículo, es sobre este último bloque, lo metodológico y específicamente sobre el abordaje etnográfico al servicio de la publicidad, que versan los siguientes apartados.

\section{- El trabajo de campo etnográfico en la investigación publicitaria}

El trabajo de campo, tal como es concebido en la etnografía, pretende

[...] recabar información y material empírico que permita especificar problemáticas teóricas (lo general en su singularidad), reconstruir la organización y la lógica propias de los grupos sociales (la perspectiva del actor como expresión de la diversidad); reformular el propio modelo teórico, a partir de la lógica reconstruida de lo social (Guber, 2004, p. 49).

La comprensión de esto es fundamental en tanto que solo es posible, desde lo que plantea la perspectiva etnográfica, recoger los datos en los contextos en los que estos se originan, pues es allí donde se recubren de significado y, a su vez, definen la manera como el contexto mismo se estructura de una manera particular. Ello implica entender que lo que hace que un fenómeno sea considerado social y cultural es que este tenga un sentido determinado por los miembros del colectivo en el que se produce, de tal manera que "El único medio para acceder a esos significados que los sujetos negocian e intercambian, emiten y reciben, es la vivencia, la posibilidad de experimentar en carne propia esos sentidos, como lo hacen todos los individuos en su socialización" (Guber, 2004, p. 111).

Por su parte, la publicidad, tal como lo plantea Colón Zayas (irradiado por la visión sociológica europea encabezada por Jean Baudrillard) "es el discurso de los objetos" (2001, p. 23), y al apoderarse de tal discurso se inserta indiscutiblemente en el mundo de las mercancías ${ }^{2}$, "las cuales [...] representan formas sociales y distribuciones de cono-

2 En lo que respecta al concepto de mercancías, estas han de entenderse en el marco de este artículo como aquellas tangibles e intangibles y, por tanto, comprenden tanto productos como servicios e ideologías. 
cimiento muy complejas" (Appadurai, 1991, p. 60). Dicho conocimiento ha de ser tanto del orden productivo como del consumo mismo, puesto que la producción responde no solo a un asunto meramente funcional, sino que también lo hace a unas preconcepciones sean estas de un orden cosmogónico, ontológico y/o deontológico que son compartidas colectivamente; y el consumo está en directa relación con las realidades sociohistóricas en las cuales se inscribe ese colectivo (Sassatelli, 2012).

Así pues, un objeto/servicio/producto será usado o "consumido" a partir de los significados que la sociedad, en la que se inscribe, le ha otorgado a dicho objeto/servicio/ producto. Esta dotación no solo funcional, sino también (y sobre todo) simbólica se hace por diferentes medios, entre los que se cuenta el mensaje publicitario, el cual sufre un proceso de descodificación al llegar al consumidor, subjetivando dicho objeto/servicio/ producto, a partir de la información socio-histórica-cultural que este tiene. De tal manera que, como lo plantea Sassatelli "La publicidad tiene [...] una abierta función ideológica en sentido lato, o sea que transmite ideas y hace circular clasificaciones culturales [...]" (Sassatelli, 2012, p. 187).

Ahora bien, es claro que para que la publicidad pueda cumplir esta función ideológica requiere necesariamente de la lecturabilidad de los contextos. Es en este lugar en el que entra la etnografía y especialmente la reflexividad etnográfica, de acuerdo con lo planteado anteriormente, dado que se convierte en método de lectura de los sujetos insertos en unas realidades sociales regladas y mediadas por unos valores subjetivados culturalmente; reglas y valores que solo pueden ser vistos en el accionar cotidiano de los grupos y que exigen para su comprensión de una interrelación de interpretaciones entre el actor y el investigador, lo que se denomina perspectivas EMIC y ETIC, respectivamente.

\section{- Lo EMIC y lo ETIC en publicidad}

Como se ha evidenciado a lo largo del texto, la intención de la etnografía reside en la explicación de los fenómenos socioculturales, reconociendo a los actores como constructores, configuradores, dotadores y, por tanto, legitimadores de significados a los eventos, prácticas, objetos y demás elementos constituyentes de su mundo accesible y no accesible. Esto significa tomar en el extremo de un continuum la acción, la percepción, la opinión, el sentido, la interpretación y la emoción -entre otras cosas- del actor; en palabras antropológicas: la Perspectiva EMIC, en tanto que "[...] el mundo social está preconstituido por marcos de sentido propios de quienes en ellos se desenvuelven [... y esos marcos existen como objetividad social" (Guber, 2004, p. 44)

En el otro extremo de dicho continuum, no en oposición, sino en complementariedad, se encuentra la denominada Perspectiva ETIC, es decir, la mirada de quien observa, de quien investiga, puesto que en virtud de los sentidos que quiere o necesita desentrañar, requiere comprender cómo se han construido esos marcos de objetividad social. En continuidad con Guber:

Para acceder a la perspectiva del actor y construirla, para relevar aspectos informales o no documentados y establecer contradicciones y relaciones entre verbalizaciones y 
prácticas, para evidenciar la articulación entre los distintos aspectos de la vida social, para ampliar y descentrar la mirada sobre los sujetos, la presencia directa en el campo (pilar de las concepciones empiristas) es condición necesaria pero no suficiente. A ello se añade, ahora, la elaboración teórica y del sentido común que, desde el principio al final, permite apropiarse de la información, transformarla en dato y organizarla en una explicación (2004, p. 43).

Tal aseveración tiene su fundamento en el hecho de que no es siempre equivalente que alguien hable de lo que piensa, siente o cree sobre algún aspecto de su realidad (u otra realidad lejana en espacio y/o tiempo), a que actúe con respecto a esa realidad, en función de sus estructuras mentales, las cuales pueden ser de carácter consciente o inconsciente y que están atravesadas por el corpus colectivo en el cual se inscriben. Esto es: existe una diferencia de marcado acento entre lo que se dice que se hace o lo que se debería hacer y lo que en realidad se hace y, solo la mirada crítica y atenta del investigador logra establecer tal diferencia.

Ahora bien ¿de qué manera este cruce e imbricamiento de perspectivas le ayuda a la publicidad?

Pues bien, se hace necesario, antes de dar respuesta a esta pregunta, o más bien como una forma de brindar un marco interpretativo, plantear que el campo en que opera la publicidad no se limita a su función comercial; cumple, en muchas ocasiones, como ya se mencionó, una función ideológica (Sassatelli, 2012), a partir de asociaciones de carácter simbólico, construidas en el discurso retórico del mismo mensaje publicitario. Obviamente, para lograr este carácter simbólico, estas asociaciones deben estar inscritas no solo sobre los significados con los que los colectivos dotan a las mercancías, sino también con la manera como dichos colectivos representan, viven, prefiguran y proyectan su entorno, sus instituciones sociales y todo lo que los conforma, define y hasta delimita: "Como agente de la práctica social, la publicidad reafirma las representaciones sociales vigentes a los/ las receptores/as de sus creaciones simbólicas" (Ellin, 2010, p. 141).

Esto no es otra cosa diferente a entender que es la cultura la se encarga de determinar y/o de dirigir las escogencias que los miembros de una sociedad deben hacer, a partir del abanico de posibilidades que se ofrecen, porque con dichas posibilidades se comunica el lugar ocupado en la sociedad a la cual el sujeto se encuentra incorporado y que, además, permite -real o imaginariamente- alcanzar lo que se quiere o desea ser: "La pertenencia y la aceptación que un individuo tiene dentro de un grupo dependen de manera considerable de la forma como se relaciona con los objetos y las ideas que consume" (Méndez, 2007, p. 300).

Es aquí donde radica la propuesta de la pesquisa publicitaria desde el cruce EMIC/ ETIC: en poder comprender cuáles son las mercancías/marcas a las cuales se les ha dotado de tal sentido simbólico, tanto desde el discurso del sujeto (sea este individual, social y/o colectivo), como desde quien investiga; y es este entrecruzamiento interpretativo el que teje el tendido sobre el cual la publicidad puede proponer una retórica en sus mensajes 
publicitarios acorde con las representaciones de realidad que dichos sujetos han establecido, a partir de sus contextos históricos, sociales, geográficos, etc.

Dicho de otra manera, se entiende que el objetivo desde la reflexividad etnográfica en la publicidad es encontrar, a manera de vasos comunicantes, la relación entre el bien objeto promocionado y la representación del mundo que tienen los colectivos. Representación que, como se ha visto a lo largo del texto, es culturalmente construida y restringe -y en algunos casos no admite- subversiones a los órdenes sociales que establece.

Si esta conexión no se realiza, coherente y conscientemente, las estrategias publicitarias seguirán siendo, en su gran mayoría, un esfuerzo fundamentado en el ensayo/ error o, lo que es peor aún, un trasplante de mensajes que, por su éxito o impacto, han logrado los posicionamientos esperados de una mercancía en una latitud hacia otra en la cual no se alcanza el mismo éxito y posicionamiento, por la variabilidad de sentidos que se pueden encontrar de un lugar a otro.

\section{Conclusiones: retos y posibilidades}

Como se ha buscado evidenciar, la reflexividad etnográfica como herramienta investigativa puede posicionarse como una de las mejores estrategias para el abordaje del consumidor, en tanto sujeto atravesado por el devenir de su realidad social, la cual está constituida por reglas y valores que le son propios y que trazan las rutas por las cuales ha de transitar. Estas reglas y valores van desde lo que atañe a las condiciones mínimas de supervivencia, hasta aquello que se convierte en generador de estatus y posición en un contexto social y cultural determinado.

Visto así, este abordaje metodológico proporciona un visión holística de ese sujeto que consume, en relación consigo mismo, con el entorno y con los objetos y/o bienes disponibles para la satisfacción de sus necesidades, en tanto que permite un análisis de los procesos mercantiles o no, de los que disponen los miembros de una sociedad que, a su vez, se convierten en configuradores de representaciones sociales e imaginarios en relación con las adquisiciones, usos y adhesiones, entre otras cosas de lo que en este texto se ha denominado como mercancías.

La posibilidad más significativa que esta herramienta le propone al ejercicio publicitario es hallar, dentro del cúmulo de datos cotidianos, los elementos comunes que subyacen en las configuraciones sociales, buscando que la retórica en este tipo de comunicación sea acorde con estas, a fin de cumplir con su condición de discurso persuasivo.

El reto descansa, si bien no en el abandono de la frenética producción publicitaria a fin de satisfacer los deseos de los anunciantes -en tanto dinámica propia del sistema económico occidental-, sí en la toma de conciencia y en la implementación cada vez más efectiva de lecturas contextualizadas de las sociedades en las cuales se pretende insertar. Se trata, entonces, de responder con la celeridad exigida pero con la reflexividad necesitada. 


\section{Bibliografía}

Ameigeiras, A. R. (2006). El abordaje etnográfico en la investigación social. En I. V. Gialdino, Estrategias de investigación cualitativa (pp. 107-151). Barcelona: Gedisa.

Appadurai, A. (1991). La vida social de las cosas. Perspectiva cultural de las mercancías. México: Grijalbo.

Bourdieu, P. (1997). Espacio social y campo de poder. Barcelona: Anagrama.

Canclini, N. G. (1995). Consumidores y ciudadanos. Conflictos multiculturales de la globalización. Mexico: Grijalbo.

Colón, E. (2001). Publicidad y hegemonía. Matrices discursivas. Bogotá: Norma.

Douglas E Isherwood. (1979). El mundo de los bienes. Hacia una antropología del consumo. Mexico: Grijalbo.

Ellin, L. (2010). Representaciones sociales a futuro en la publicidad. Bakhtiniana, 140-152.

Gil, Gil, López, Pineda E Zapata. (2007). Sistematización proyecto redes pedagógicas Medellín 2004-2007. Redes pedagógicas, construcción desde el desarrollo humano: un laberinto donde aventurarse. En Antioquia, Redes pedagógicas. Experiencias con sentido.. (p.s. 55-98). Medellín: Editorial Universidad de Antioquia.

Guber, R. (2001). La etnografía. Método, campo y reflexividad. Bogotá: Norma.

Guber, R. (2004). El salvaje metropolitano. Reconstrucción del conocimiento social en el trabajo de campo. Buenos Aires: Paidós.

Malinowski, B. (2001). Los argonautas del Pacífico occidental. Barcelona: Península S. A.

Méndez, C. (2007). Comunicación e identidad: una aproximación al estudio del consumo. Universitas Humanística, 291-305.

Moyano, C. M. (2002). Comportamiento del consumidor: valores culturales de la población de Montevideo y Gran Montevideo. IMUR, 3-14.

Rivas, J. A. (2001). Comportamiento del consumidor. Madrid: ESIC.

Rivera, Arellano \& Molero. (2000). Conducta del consumidor. Estrategias y técnicas aplicadas al marketing. Madrid: ESIC.

Sánchez, J. R. (1993). Teoría de la publicidad. Madrid: Tecnos.

Sassatelli, R. (2012). Consumo, cultura y sociedad. Buenos Aires: Amorrortu.

Schiffman E Kanuk. (1997). Comportamiento del consumidor. México: Prentice-Hall Hispanoamericana S. A.

Solomon, M. (2008). Comportamiento del consumidor. México: Prentice Hall. 